\title{
A global fit of top quark effective theory to data
}

Andy Buckley, Christoph Englert, James Ferrando, David J. Miller, Liam Moore, Michael Russell, and Chris D. White SUPA, School of Physics and Astronomy, University of Glasgow, Glasgow, G12 8QQ, UK

In this paper we present a global fit of beyond the Standard Model (BSM) dimension six operators relevant to the top quark sector to all currently available top production cross-section measurements, namely parton-level top-pair and single top production at the LHC and the Tevatron. Higher order QCD corrections are modelled using differential and global $K$-factors, and we use novel fast-fitting techniques developed in the context of Monte Carlo event generator tuning to perform the fit. This allows us to provide new, fully correlated and model-independent bounds on new physics effects in the top sector from the most current direct hadron-collider measurements in light of the involved theoretical and experimental systematics. As a by-product, our analysis constitutes a proof-of-principle that fast fitting of theory to data is possible in the top quark sector, and paves the way for a more detailed analysis including top quark decays, detector corrections and precision observables.

\section{INTRODUCTION}

The Standard Model of particle physics has proven to be an extremely successful description of Nature up to the electroweak scale. Nonetheless there are many compelling reasons to believe it is an intermediate step to a more fundamental picture of physics at the $\mathrm{TeV}$ scale.

The top quark, as the heaviest Standard Model particle, is expected to play a unique role in this new physics. Given the unsatisfactory explanation of electroweak symmetry breaking within the SM and the appearance of $m_{t}$ at the electroweak scale, i.e. the closeness of the top Yukawa coupling to unity, the top mass may arguably be seen as a strong hint of physics beyond the SM.

Most BSM scenarios lend a special role to the top quark. In supersymmetry the light Higgs mass is stabilised from UV divergences by the contribution of SUSY top partners, among others (see, e.g., Refs. [1, 2]). In compositeness scenarios [3, 4, the quark masses and EWSB are generated through linear couplings of the SM fermions to new strongly-interacting physics at the $\mathrm{TeV}$ scale. In theories of warped extra-dimensions, the top quark couples preferentially to Kaluza-Klein states in the 5D bulk [5, 6], offering a unique window to the new physics.

Typically all these scenarios predict a modification of Higgs phenomenology, which has been thoroughly studied after the Higgs discovery 7-11]. Such analyses are currently limited by small statistics in the observed Higgs discovery modes. Taking the special role of the top quark in electroweak symmetry breaking at face value, the abundant production of top quarks at the LHC provides a complementary avenue to search for new non-resonant physics beyond the SM, that will be relevant to our understanding of electroweak symmetry breaking.

Given the plethora of concrete scenarios and the absence of any telling signals of new physics in the current data, parametrising BSM effects in an effective field theory expansion [23] is well-motivated. In this approach, all possible interactions are captured in an effective La- grangian $\mathcal{L}_{\text {eff }}$ :

$$
\mathcal{L}_{\text {eff }}=\mathcal{L}_{\mathrm{SM}}+\frac{1}{\Lambda} \mathcal{L}_{1}+\frac{1}{\Lambda^{2}} \mathcal{L}_{2}+\ldots .
$$

The higher-dimensional Lagrangian terms $\mathcal{L}_{i}$ are suppressed by powers of $\Lambda$ - the energy scale associated with the new physics. In the top-down approach, we have integrated out all heavy degrees of freedom, capturing their low energy phenomenology guided by SM gauge and global symmetries, irrespective of their concrete UV dynamics. Such an expansion is valid provided there is a good separation of scales between the typical collider energy and $\Lambda$. However, this approach is completely general: the $\left\{\mathcal{L}_{i}\right\}$ are constructed from SM operators, respecting the $S U(3) \times S U(2) \times U(1)$ gauge symmetry.

The leading contributions relevant to new physics in the top sector enter at the dimension-six level $\mathcal{O}\left(1 / \Lambda^{2}\right)$

$$
\mathcal{L}_{\text {eff }}=\mathcal{L}_{\mathrm{SM}}+\frac{1}{\Lambda^{2}} \sum_{i} C_{i} O_{i}+\mathcal{O}\left(\Lambda^{-4}\right),
$$

where $C_{i}$ are arbitrary 'Wilson coefficients' and $O_{i}$ are

\begin{tabular}{lll}
\hline 4-fermion operators & Non 4-fermion operators \\
\hline$O_{q q}^{1}\left(\bar{q} \gamma_{\mu} q\right)\left(\bar{q} \gamma^{\mu} q\right)$ & $O_{\phi q}^{3}$ & $i\left(\phi^{\dagger} \tau^{I} D_{\mu} \phi\right)\left(\bar{q} \gamma^{\mu} \tau^{I} q\right)$ \\
$O_{q q}^{3}\left(\bar{q} \gamma_{\mu} \tau^{I} q\right)\left(\bar{q} \gamma^{\mu} \tau^{I} q\right)$ & $O_{t W}\left(\bar{q} \sigma^{\mu \nu} \tau^{I} t\right) \tilde{\phi} W_{\mu \nu}^{I}$ \\
$O_{u u}\left(\bar{u} \gamma_{\mu} u\right)\left(\bar{u} \gamma^{\mu} u\right)$ & $O_{t G}\left(\bar{q} \sigma^{\mu \nu} \lambda^{A} t\right) \tilde{\phi} G_{\mu \nu}^{A}$ \\
$O_{q u}^{8}\left(\bar{q} \gamma_{\mu} T^{A} q\right)\left(\bar{u} \gamma^{\mu} T^{A} u\right)$ & $O_{G}$ & $f_{A B C} G_{\mu}^{A \nu} G_{\nu}^{B \lambda} G_{\lambda}^{C \mu}$ \\
$O_{q d}^{8}\left(\bar{q} \gamma_{\mu} T^{A} q\right)\left(\bar{d} \gamma^{\mu} T^{A} d\right)$ & $O_{\tilde{G}} f_{A B C} \tilde{G}_{\mu}^{A \nu} G_{\nu}^{B \lambda} G_{\lambda}^{C}$ \\
$O_{u d}^{8}\left(\bar{u} \gamma_{\mu} T^{A} u\right)\left(\bar{d} \gamma^{\mu} T^{A} d\right)$ & $O_{\phi G}\left(\phi^{\dagger} \phi\right) G_{\mu \nu}^{A} G^{A \mu \nu}$ \\
& $O_{\phi \tilde{G}}\left(\phi^{\dagger} \phi\right) \tilde{G}_{\mu \nu}^{A} G^{A \mu \nu}$ \\
\hline
\end{tabular}

TABLE I: All dimension-six operators relevant to top quark production, in the notation of Ref. [12. Details of each are included in the text. $q$ denotes the left-handed quark doublet, $u$ and $d$ denote the up-type and down-type right-handed singlets. We do not include explicit flavor indices here, the relevant flavor indices are included in the text. 13 operators are shown, but $O_{t W}$ and $O_{t G}$ have both real and imaginary parts which should be considered as independent operators; the latter produce $\mathcal{C} \mathcal{P}$-violating effects. 


\begin{tabular}{|c|c|c|c|}
\hline Dataset & $\sqrt{s}(\mathrm{TeV})$ & Measurements & Ref. \\
\hline \multicolumn{4}{|c|}{ Top pair production } \\
\hline \multirow[t]{2}{*}{ ATLAS } & $7+8$ & Total inclusive $\sigma$ & 13 \\
\hline & $7+8$ & Differential $p_{T}(t), M_{t \bar{t}},|y(t \bar{t})|$ & 14 \\
\hline CMS & 7 & Differential $p_{T}(t), M_{t \bar{t}}, y(t),|y(t \bar{t})|$ & 15 \\
\hline $\mathrm{CDF}$ & 1.96 & Differential $M_{t \bar{t}}$ & 16 \\
\hline $\mathrm{D} \emptyset$ & 1.96 & Differential $M_{t \bar{t}}, p_{T}(t),|y(t)|$ & 17 \\
\hline \multicolumn{4}{|c|}{ Single top production } \\
\hline \multirow{2}{*}{ ATLAS $t$-channel } & 7 & Total inclusive $\sigma$ & \\
\hline & 7 & Differential $p_{T}(t),|y(t)|$ & \\
\hline \multirow[t]{2}{*}{ CMS $t$-channel } & 7 & Total inclusive $\sigma$ & 19 \\
\hline & 8 & Total inclusive $\sigma$ & 20 \\
\hline CDF s-channel & 1.96 & Total inclusive $\sigma$ & 21 \\
\hline $\mathrm{D} \emptyset s+t$-channel & 1.96 & Total inclusive $\sigma$ & 22 \\
\hline
\end{tabular}

TABLE II: Datasets used in the fit, including total cross-sections $(\sigma)$; transverse momenta of single tops $\left(p_{T}(t)\right)$ and top pairs $\left(p_{T}(t \bar{t})\right)$; rapidities of single tops $(y(t))$ and top pairs $(y(t \bar{t}))$; and the invariant mass of top pairs $\left(M_{t \bar{t}}\right)$. dimension-six operators. These operators lead to noticeable deviations from SM expectations in a double expansion of the matrix element in SM and new physics couplings

$$
\left|\mathcal{M}_{\text {tot }}\right|^{2}=\left|\mathcal{M}_{\mathrm{SM}}\right|^{2}+2 \Re\left\{\mathcal{M}_{\mathrm{SM}} \mathcal{M}_{D 6}^{*}\right\}+\left|\mathcal{M}_{D 6}\right|^{2},
$$

where strictly speaking one must neglect the third term on the right-hand side if working to dimension-six only, as this has dimension-eight, . Provided $C_{i} / \Lambda^{2}$ is small, such a truncation is typically valid and the squared dimensionsix terms become numerically irrelevant.

The complete set of 80 effective operators at dimensionsix has been known for some time [24 26]. Only recently was it shown that this basis contains several redundancies, with the minimal set comprising 59 terms [12, 27, 28, 28]. Considerable attention has been devoted to constraining these operators, for example, in the context of Higgs and precision electroweak physics [7-11. In addition, strong bounds have also been placed on new top interactions from precision constraints at LEP [29] and direct searches for top quark physics at the LHC 3035 .

While Higgs physics has received a lot of attention from an EFT perspective, the top quark sector has not seen similar scrutiny, although top data from the combination of the Tevatron and the LHC Run I is far more abundant. In the last few years, top quark physics has entered something of a precision era: the top has been measured in several production and decay channels, and dedicated searches in complicated final states such as $t \bar{t} H$ are underway [36, 37.

It is our aim to close this gap. The TopFITTER approach constrains new physics in the top sector using both differential and inclusive observables, by means of a computational tool which is fully flexible with respect to the number of input measurements and scales well to the relevant number of EFT operators. In the present work we limit ourselves to a nine-dimensional fit based on direct top measurements performed at the Tevatron and the LHC, keeping track of all EFT operator-correlations, and reserve a more complete investigation for the near future 38 .

\section{RELEVANT OPERATORS}

Throughout the analysis, and for ease of comparison with precision electroweak studies, the operator set presented in Ref. 12 is used (see also the basis of Refs. [39, 40]). Assuming minimal flavor violation, and in the leading-order* approximation of equation 2, of these 59 operators only 15 - shown in Table $\mathrm{I}$ - are relevant for top production. Fitting a 15-dimensional function is a considerable challenge; a brute force likelihood scan at $N$ points per dimension would require $N^{15}$ evaluations, which is prohibitive even for modest, low-resolution values of $N$. This naïve dimensionality can be reduced, however, by noting some features of the operator set.

Firstly, we note that the two operators containing the dual field-strength tensor $\tilde{G}_{\mu \nu}=\epsilon_{\mu \nu \rho \sigma} G^{\rho \sigma}$, along with the imaginary parts of $O_{t G}$ and $O_{t W}$, are $\mathcal{C P}$-odd and can be discriminated from $\mathcal{C P}$-even effects in studies of spin-correlations, polarisation effects and genuinely $\mathcal{C P}$ sensitive observables [42] (for recent analyses focusing on the $t W b$ vertex, for instance, see Refs. [43 47]). Currently there is no evidence for $\mathcal{C P}$-violation in the top sector beyond the minimal flavor violation assumption. We will address these operators in forthcoming work but neglect them in the following; the dimensionality of our fit is reduced by four.

Secondly, we consider top-pair production. Here the four-fermion operators, which are numerous when all flavour combinations are considered, only contribute to

\footnotetext{
*B By leading-order we mean $\mathcal{O}\left(\Lambda^{-2}\right)$, but for some new physics effects, such as top flavour-changing neutral currents, the first nonzero contributions enter at $\mathcal{O}\left(\Lambda^{-4}\right)$ see e.g. 41 for details.
} 
top-pair production through the partonic subprocesses $u \bar{u}, d \bar{d} \rightarrow t \bar{t}$, which reduces the myriad of possible operators to four unique, flavour-specific linear combinations 39, 48:

$$
\begin{aligned}
C_{u}^{1}= & 3\left(2 C_{q q}^{(1) 1331}+C_{u u}^{1331}\right) \\
& -\left(C_{q q}^{(1) 1133}+C_{q q}^{(3) 1133}+C_{u u}^{1133}\right) \\
C_{u}^{2}= & -\left(C_{q u}^{(8) 1133}+C_{q u}^{(8) 3311}\right) \\
C_{d}^{1}= & 3\left(C_{q q}^{(3) 1331}-C_{q q}^{(1) 1331}\right) \\
& +\left(C_{q q}^{(3) 1133}-C_{q q}^{(1) 1133}\right)+6 C_{u d}^{(8) 3311} \\
C_{d}^{2}= & -\left(C_{q u}^{(8) 1133}+C_{q d}^{(8) 3311}\right),
\end{aligned}
$$

where explicit flavor indices $\left(\bar{q}^{i} q^{j}\right)\left(\bar{q}^{j} q^{k}\right)$ have now been included. The non-4-fermion operators $O_{G}, O_{t G}$, and $O_{\phi G}$ also contribute to top pair production, giving a total of 7 relevant operators. In the $g g \rightarrow t \bar{t}$ channel, $O_{G}$ rescales the triple gluon vertex while $O_{t G}$ modifies the top-gluon coupling; $O_{\phi G}$ only contributes through $g g \rightarrow h \rightarrow t \bar{t}$, which is heavily suppressed in the Standard Model although it can be probed in $t t H$ production.

Three $\mathcal{C} \mathcal{P}$-even operators ${ }^{\dagger}$ contribute to single top production: $O_{t W}$ modifies the $t W b$ vertex, as does $O_{\phi q}^{3}$, while the operator $O_{q q}^{(3)} 1331$ creates a new four-quark topology which interferes with the SM piece.

There is hence a clean factorisation into $7+3 \mathcal{C P}$-even operators associated with top quark production at hadron colliders. In this study we reduce this further to a $6+3$ configuration by eliding the highly suppressed contributions of $O_{\phi G}$ to top-pair production.

\section{DATASETS}

The aim of this paper is to present a preliminary study demonstrating the feasibility of performing a full global fit of top quark effective theory to data. We thus include top quark pair and single top production processes at parton level only, which observables and datasets [14 22 are collected in Table II

It should be noted that a fully differential fit along these lines consists of a multitude of exclusive measurements. Treating each bin as an independent ${ }^{\ddagger}$ measurement, we have 103 bins for top pair production and 23 from single top. This highlights the necessity of a fast analysis framework, as introduced in the present work.

Given that we will model higher-order corrections as described in the following section, we do not include $W t$ production, which interferes with top pair production

\footnotetext{
${ }^{\dagger}$ The contribution of the operator $O_{\phi q}^{1}=\left(\phi^{\dagger} D_{\mu} \phi\right)\left(\bar{t} \gamma^{\mu} b\right)$ is heavily suppressed, as its interference with the SM amplitude is proportional to $m_{b}$ (see e.g. 49 ).

${ }^{\ddagger}$ Where published by the experiments, we have included bin-tobin correlations. These have negligible effect on our conclusions.
}

at next-to leading order, such that it is not possible to reproduce existing experimental analyses using a fixed order parton level calculation 50,54 .

\section{DETAILS OF ANALYSIS}

We begin by including the operators listed above (together with consequent SM parameter redefinitions) in a FEYNRULES 55] model file, which is then interfaced via UFO [56] to MADGRAPH/MAdEvent [56, 57] in order to obtain parton-level theory predictions. Samples were generated for all the relevant processes: top pair production: $p p \rightarrow t \bar{t}$, single top production: $p p \rightarrow t \bar{b}$ (s-channel), and $p p \rightarrow t q$ ( $t$-channel)

In order to model next-to leading order QCD corrections, SM-only samples at next-to-leading order are generated with MCFM [58. These are used to construct differential (bin-by-bin) and global $K$-factors, as in e.g. Ref. [59]. Theoretical uncertainties for these samples are estimated in the usual way, by independently varying the scales $\mu_{\text {central }} / 2<\mu_{\mathrm{R}, \mathrm{F}}<2 \mu_{\text {central }}$, where $\mu_{\text {central }}$ is taken to be $m_{t}$. Parton distribution function (PDF) uncertainties are estimated by generating events using the next-to-leading order NNPDF23 [60, MSTW2008 61], and CT10 62 PDF sets, according to the PDF4LHC 63] prescription. We take the central value as our estimate and the width of the envelope (including scale variations) as the total theoretical uncertainty. In the case of top pair total inclusive cross-sections, we use global $K$-factors from next-to-next-to leading order QCD with soft gluons resummed to next-to-next-to-leading logarithmic accuracy 6468 .

A strength of our fitting procedure is the use of novel techniques developed in the context of Monte Carlo event generator tuning, as implemented in the Professor 69 framework. The procedure is as follows:

- A set of points in the $N$-dimensional parameter space $\left\{C_{i}\right\}$ is sampled logarithmically. Other samplings are possible - we choose logarithmic sampling to avoid oversampling of regions where coefficients are large, such that dimension-eight terms become important.

- At each sampled parameter space point, all theory observables are calculated, with uncertainties, as described above. One then constructs a polynomial parameterising function $f_{b}\left(\left\{C_{i}\right\}\right)$ for each observable bin $b$, which fits the sampled points with least-squares-optimal precision. This function can be used to efficiently generate theory predictions for arbitrary parameter space points within the fitted range. We choose a third-degree polynomial for this function. This has been shown to work well in Monte Carlo tuning [69], and should in fact be better suited to the present case: in the absence of uncertainties, each observable is a second-order 


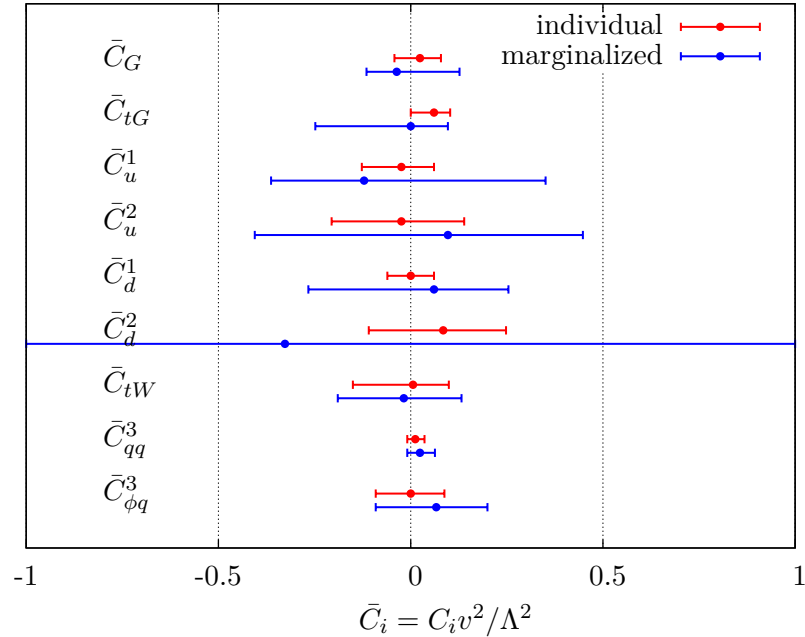

FIG. 1: 95\% confidence intervals for operators contributing to top-pair and single top production, individually (with all other operators set to zero) and marginalised (with all other operators allowed to float to best-fit values). Note that the marginalised bound on $\bar{C}_{d}^{2}$ fall outside the region where the dimension-six approximation is valid, so this operator is unconstrained.

polynomial in the $\left\{C_{i}\right\}$, cf. eq. (1), and the extra polynomial order provides some tolerance to beyond-fixed-order effects.

- Finally, we construct a $\chi^{2}$ function between the bin parameterisations $\left\{f_{b}\left(\left\{C_{i}\right\}\right)\right\}$ and the data, according to

$$
\chi^{2}\left(\left\{C_{i}\right\}\right)=\sum_{\mathcal{O}} \sum_{b} \frac{\left(f_{b}\left(\left\{C_{i}\right\}\right)-E_{b}\right)^{2}}{\sigma_{b}^{2}},
$$

i.e. we sum over all observables $\mathcal{O}$, and all bins in that observable, $b . E_{b}$ is the experimental reference value at bin $b$ and $\sigma_{b}$ is the total uncertainty for bin $b$, which we for now assume as an uncorrelated combination of theoretical modelling and experimental measurement uncertainties, $\sigma_{b}=\sqrt{\sigma_{\text {theory }}^{2}+\sigma_{\exp }^{2}}$. The $\chi^{2}$ is then used to place constraints on the operator Wilson coefficients, as follows.

Constraints are obtained in two ways, for ease of comparison with existing literature. Firstly, single operator coefficients are allowed to vary, with all others set to zero (the SM value). The $\chi^{2}$ is then minimised using PyMinuit 70, and used to set confidence limits on the operator value. A second approach is to marginalise over the remaining operators, namely to construct the confidence limit for a given operator coefficient whilst allowing all other coefficients to vary. Both cases are shown in Figure 1. where the dimension-six contributions are normalised to the Standard Model piece via $\bar{C}_{i}=C_{i} v^{2} / \Lambda^{2}$. All results are consistent with the SM within $95 \%$ limits.
As with all effective operator constraints, these must be interpreted as valid only in the region where $\mathcal{O}\left(\Lambda^{-4}\right)$ terms are not large. Clearly $\bar{C}_{d}^{2}$ is outside this region. In top pair production, for instance, the contribution from dimension- 6 operators relative to the SM piece is typically $\mathcal{O}\left(g_{s}^{2} C_{i} v^{2} / \Lambda^{2}\right)$ which must be $<1$ in the linear approximation, i.e $\bar{C}_{i} \lesssim 1.5$. All other operators respect this bound. It should be noted that some of these operators, namely those containing field strength tensors, can only be generated at loop level in the ultraviolet completion, which widens this region of validity since $\Lambda^{2}$ will be accompanied by a loop factor of $16 \pi^{2}$. This argument is invalid, however, if the underlying completion is strongly coupled. It is possible to include such information in our fitting approach, but in the interests of full generality no such model-specific assumptions are made here.

One sees from Figure 1 that the weakest constraints are on the coefficients (of four-fermion operators) $\bar{C}_{u}^{i}$ and $\bar{C}_{d}^{i}$. These are constrained by the processes $u \bar{u} \rightarrow t \bar{t}$ and $d \bar{d} \rightarrow t \bar{t}$ respectively, which are suppressed relative to the corresponding gluon initiated processes, mostly due to the relative partonic luminosities.

One may also examine the correlation of constraints between pairs of operators. An example is Figure 2(a), which shows confidence limits in the $\left(C_{t W}, C_{\phi q}^{3}\right)$ plane, with all other operator coefficients set to zero. One may also marginalise over all remaining operators, as shown in Figure 2(b), In both cases, we currently find excellent agreement with the SM. More detailed results will be presented in a forthcoming paper 38].

\section{SUMMARY, CONCLUSIONS AND OUTLOOK}

Following the discovery of the Higgs boson, the search for physics beyond the Standard Model will remain the primary goal of the LHC experiment for the foreseeable future. The top quark sector is a particularly well-motivated window through which to look for the imprint of nonresonant new physics. Modelling such effects using effective field theory (higher dimensional operators) is well justified given the absence of new resonant physics from the LHC Run I. The abundance of top quark production at the LHC enables a multi-faceted analysis of top quark phenomenology and allows us to confront higher dimensional top sector operators with differential measurements at high statistics.

In this paper, we have characterised new physics corrections using the well-established framework of effective field theory. We have presented results from a new computational framework to fit all possible dimension-six operator coefficients to a comprehensive set of relevant data. This is possible through our use of fast-fitting algorithms, which have been developed (and well-tested) in the context of Monte Carlo event generator tuning. Here we expect these techniques to work even better, given the explicit polynomial dependence of theory observables on 


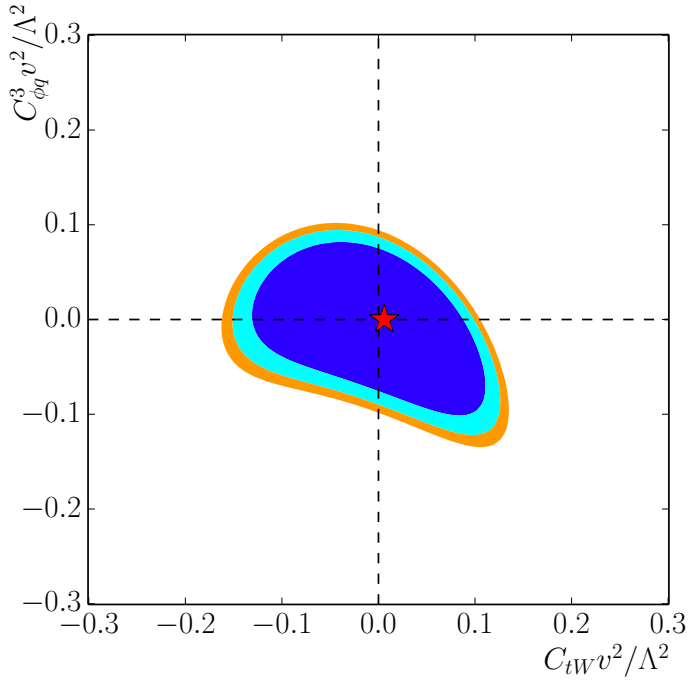

(a) individual constraints.

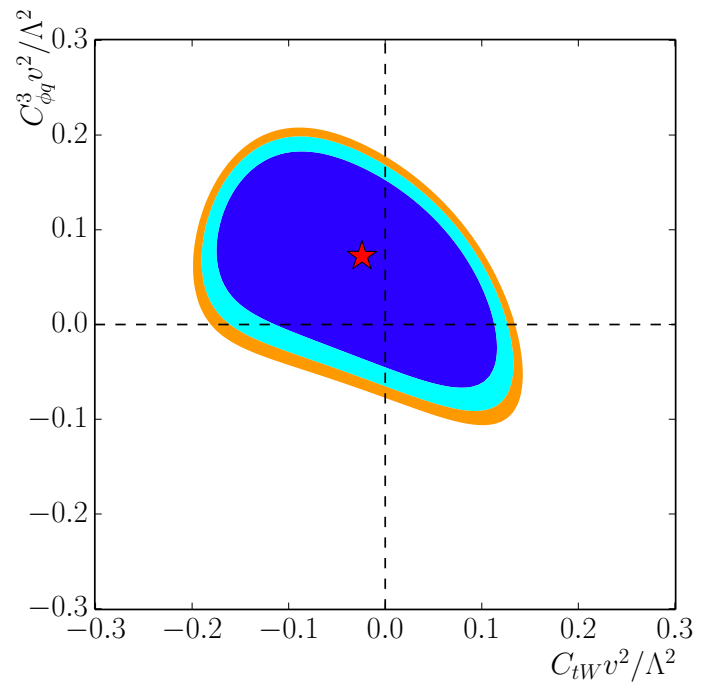

(b) marginalised constraints.

FIG. 2: $68 \%$ (blue), $95 \%$ (turquoise) and $99 \%$ (orange) confidence intervals for $C_{t W}$ and $C_{\phi q}^{3}$ in a global fit, with all remaining coefficients set to zero (a) and marginalised over (b). The star marks the best fit point, indicating a currently good agreement with the Standard Model.

operator coefficients.

Our method involves constructing a parameterising function to effectively parametrise the theory output of Monte Carlo generators (here at parton level only). Once this has been constructed, it is quick to perform a global fit containing all possible operators, and to amend this fit as and when new data appear. Furthermore, there is no significant speed decrease in our fitting procedure upon improving the theory prediction (e.g. to include parton shower or detector corrections), as such improvements only affect the parameterising function, which has to be calculated only once.

The results of our fit currently show good agreement with the Standard Model, which is unsurprising given the absence of new physics currently reported in other studies. Our results, however, provide a proof of princi- ple study that efficient global fits of top quark effective theory are possible. It is straightforward to generalise our fit to include more experimental observables (beyond parton level, including top quark decays), to improve the theory description with higher order corrections, and to include new data sets including those from the recently commenced LHC Run II. Work in these directions is ongoing.

Acknowledgements - AB is supported by a Royal Society University Research Fellowship. CE is supported by the IPPP Associateship programme. DJM, LM, MR and CDW are supported by the UK Science and Technology Facilities Council (STFC) under grant ST/L000446/1. JF is supported under STFC grant ST/K001205/1.
[1] H. P. Nilles, Phys.Rept. 110, 1 (1984).

[2] H. E. Haber and G. L. Kane, Phys.Rept. 117, 75 (1985).

[3] R. Contino, Y. Nomura, and A. Pomarol, Nucl.Phys. B671, 148 (2003), hep-ph/0306259.

[4] K. Agashe, R. Contino, and A. Pomarol, Nucl.Phys. B719, 165 (2005), hep-ph/0412089.

[5] N. Arkani-Hamed and M. Schmaltz, Phys.Rev. D61, 033005 (2000), hep-ph/9903417.

[6] T. Gherghetta and A. Pomarol, Nucl.Phys. B586, 141 (2000), hep-ph/0003129.

[7] T. Corbett, O. J. P. Eboli, D. Goncalves, J. GonzalezFraile, T. Plehn, et al. (2015), 1505.05516.

[8] J. Ellis, V. Sanz, and T. You, JHEP 1503, 157 (2015),
1410.7703 .

[9] R. Contino, M. Ghezzi, C. Grojean, M. Muhlleitner, and M. Spira, 1307, 035 (2013), 1303.3876.

[10] A. Pomarol and F. Riva, JHEP 1401, 151 (2014), 1308.2803.

[11] A. Efrati, A. Falkowski, and Y. Soreq (2015), 1503.07872.

[12] B. Grzadkowski, M. Iskrzynski, M. Misiak, and J. Rosiek, JHEP 1010, 085 (2010), 1008.4884.

[13] G. Aad et al. (ATLAS Collaboration), Eur.Phys.J. C74, 3109 (2014), 1406.5375.

[14] G. Aad et al. (ATLAS Collaboration), Phys.Rev. D90, 072004 (2014), 1407.0371.

[15] S. Chatrchyan et al. (CMS Collaboration), Eur.Phys.J. 
C73, 2339 (2013), 1211.2220.

[16] T. Aaltonen et al. (CDF Collaboration), Phys.Rev.Lett. 102, 222003 (2009), 0903.2850.

[17] V. M. Abazov et al. (D0 Collaboration), Phys.Rev. D90, 092006 (2014), 1401.5785.

[18] G. Aad et al. (ATLAS Collaboration) (2014), 1406.7844.

[19] S. Chatrchyan et al. (CMS Collaboration), JHEP 1212, 035 (2012), 1209.4533.

[20] V. Khachatryan et al. (CMS Collaboration), JHEP 1406, 090 (2014), 1403.7366.

[21] T. A. Aaltonen et al. (CDF Collaboration), Phys.Rev.Lett. 112, 231804 (2014), 1402.0484.

[22] V. M. Abazov et al. (D0 Collaboration), Phys.Lett. B726, 656 (2013), 1307.0731.

[23] S. Weinberg, Physica 96A, 327 (1979).

[24] W. Buchmuller and D. Wyler, Nucl. Phys. B268, 621 (1986).

[25] C. J. C. Burgess and H. J. Schnitzer, Nucl. Phys. B228, 464 (1983).

[26] C. N. Leung, S. T. Love, and S. Rao, Z. Phys. C 31 (1986).

[27] B. Grzadkowski, Z. Hioki, K. Ohkuma, and J. Wudka, Nucl. Phys. B689, 108 (2004), hep-ph/0310159.

[28] J. A. Aguilar-Saavedra, Nucl. Phys. B812, 181 (2009), 0811.3842 .

[29] H. Mebane, N. Greiner, C. Zhang, and S. Willenbrock, Phys.Rev. D88, 015028 (2013), 1306.3380.

[30] Z. Hioki and K. Ohkuma, Eur. Phys. J. C65, 127 (2010), 0910.3049

[31] Z. Hioki and K. Ohkuma, Eur. Phys. J. C71, 1535 (2011), 1011.2655.

[32] C. Degrande, J.-M. Gerard, C. Grojean, F. Maltoni, and G. Servant, JHEP 1103, 125 (2011), 1010.6304.

[33] C. Englert, A. Freitas, M. Spira, and P. M. Zerwas, Phys.Lett. B721, 261 (2013), 1210.2570.

[34] Z. Hioki and K. Ohkuma, Phys. Rev. D88, 017503 (2013), 1306.5387

[35] J. A. Aguilar-Saavedra, B. Fuks, and M. L. Mangano, Phys. Rev. D91, 094021 (2015), 1412.6654.

[36] V. Khachatryan et al. (CMS), Eur.Phys.J. C75, 251 (2015), 1502.02485.

[37] G. Aad et al. (ATLAS) (2015), 1503.05066.

[38] A. Buckley et al. (TopFitter), to appear (2015).

[39] S. Willenbrock and C. Zhang, Phys. Rev. D83, 034006 (2011), hep-ph/1008.3869.

[40] N. Greiner, S. Willenbrock, and C. Zhang, Phys. Lett. B704, 218 (2011), hep-ph/1104.3122.

[41] J. A. Aguilar-Saavedra, PoS ICHEP2010, 378 (2010), 1008.3225 .

[42] T. Han and Y. Li, Phys.Lett. B683, 278 (2010), 0911.2933

[43] C.-R. Chen, F. Larios, and C. P. Yuan, Phys. Lett. B631, 126 (2005), [AIP Conf. Proc.792,591(2005)], hep$\mathrm{ph} / 0503040$.

[44] J. A. Aguilar-Saavedra, Nucl. Phys. B804, 160 (2008), 0803.3810.
[45] J. A. Aguilar-Saavedra and J. Bernabeu, Nucl. Phys. B840, 349 (2010), 1005.5382.

[46] J. A. Aguilar-Saavedra, N. F. Castro, and A. Onofre, Phys. Rev. D83, 117301 (2011), 1105.0117.

[47] C. Bernardo, N. F. Castro, M. C. N. Fiolhais, H. Gonalves, A. G. C. Guerra, et al., Phys.Rev. D90, 113007 (2014), 1408.7063

[48] J. A. Aguilar-Saavedra, Nucl. Phys. B843, 638 (2011), hep-ph/1008.3562.

[49] Q.-H. Cao, J. Wudka, and C. P. Yuan, Phys. Lett. B658, 50 (2007), 0704.2809.

[50] S. Zhu (2001), hep-ph/0109269.

[51] N. Kauer and D. Zeppenfeld, Phys.Rev. D65, 014021 (2002), hep-ph/0107181.

[52] J. M. Campbell and F. Tramontano, Nucl.Phys. B726, 109 (2005), hep-ph/0506289.

[53] S. Frixione, E. Laenen, P. Motylinski, B. R. Webber, and C. D. White, JHEP 0807, 029 (2008), 0805.3067.

[54] C. D. White, S. Frixione, E. Laenen, and F. Maltoni, JHEP 0911, 074 (2009), 0908.0631.

[55] N. D. Christensen and C. Duhr, Comput.Phys.Commun. 180, 1614 (2009), 0806.4194.

[56] C. Degrande, C. Duhr, B. Fuks, D. Grellscheid, O. Mattelaer, et al., Comput.Phys.Commun. 183, 1201 (2012), 1108.2040 .

[57] J. Alwall, M. Herquet, F. Maltoni, O. Mattelaer, and T. Stelzer, JHEP 1106, 128 (2011), 1106.0522.

[58] J. M. Campbell and R. Ellis, Nucl.Phys.Proc.Suppl. 205206, 10 (2010), 1007.3492.

[59] C. Englert, D. Goncalves, and M. Spannowsky, Phys.Rev. D89, 074038 (2014), 1401.1502.

[60] R. D. Ball, L. Del Debbio, S. Forte, A. Guffanti, J. I. Latorre, et al., Nucl.Phys. B838, 136 (2010), 1002.4407.

[61] A. Martin, W. Stirling, R. Thorne, and G. Watt, Eur.Phys.J. C63, 189 (2009), 0901.0002.

[62] P. M. Nadolsky, H.-L. Lai, Q.-H. Cao, J. Huston, J. Pumplin, et al., Phys.Rev. D78, 013004 (2008), 0802.0007.

[63] M. Botje, J. Butterworth, A. Cooper-Sarkar, A. de Roeck, J. Feltesse, et al. (2011), 1101.0538.

[64] M. Beneke, P. Falgari, S. Klein, and C. Schwinn, Nucl.Phys. B855, 695 (2012), 1109.1536.

[65] M. Cacciari, M. Czakon, M. Mangano, A. Mitov, and P. Nason, Phys.Lett. B710, 612 (2012), 1111.5869.

[66] M. Czakon and A. Mitov, Comput.Phys.Commun. 185, 2930 (2014), 1112.5675.

[67] P. Bärnreuther, M. Czakon, and A. Mitov, Phys.Rev.Lett. 109, 132001 (2012), 1204.5201.

[68] M. Czakon, P. Fiedler, and A. Mitov, Phys.Rev.Lett. 110, 252004 (2013), 1303.6254.

[69] A. Buckley, H. Hoeth, H. Lacker, H. Schulz, and J. E. von Seggern, Eur.Phys.J. C65, 331 (2010), 0907.2973.

[70] F. James and M. Roos, Comput.Phys.Commun. 10, 343 (1975). 